This item was submitted to Loughborough's Research Repository by the author.

Items in Figshare are protected by copyright, with all rights reserved, unless otherwise indicated.

\title{
Use of multiobjective genetic algorithms to optimize inter-vehicle active suspensions
}

\section{PLEASE CITE THE PUBLISHED VERSION}

PUBLISHER

(C) Professional Engineering Publishing

\section{VERSION}

VoR (Version of Record)

\section{LICENCE}

CC BY-NC-ND 4.0

\section{REPOSITORY RECORD}

Mei, Tian Xiang, and Roger M. Goodall. 2019. "Use of Multiobjective Genetic Algorithms to Optimize Intervehicle Active Suspensions". figshare. https://hdl.handle.net/2134/4522. 
This item was submitted to Loughborough's Institutional Repository (https://dspace.lboro.ac.uk/) by the author and is made available under the following Creative Commons Licence conditions.

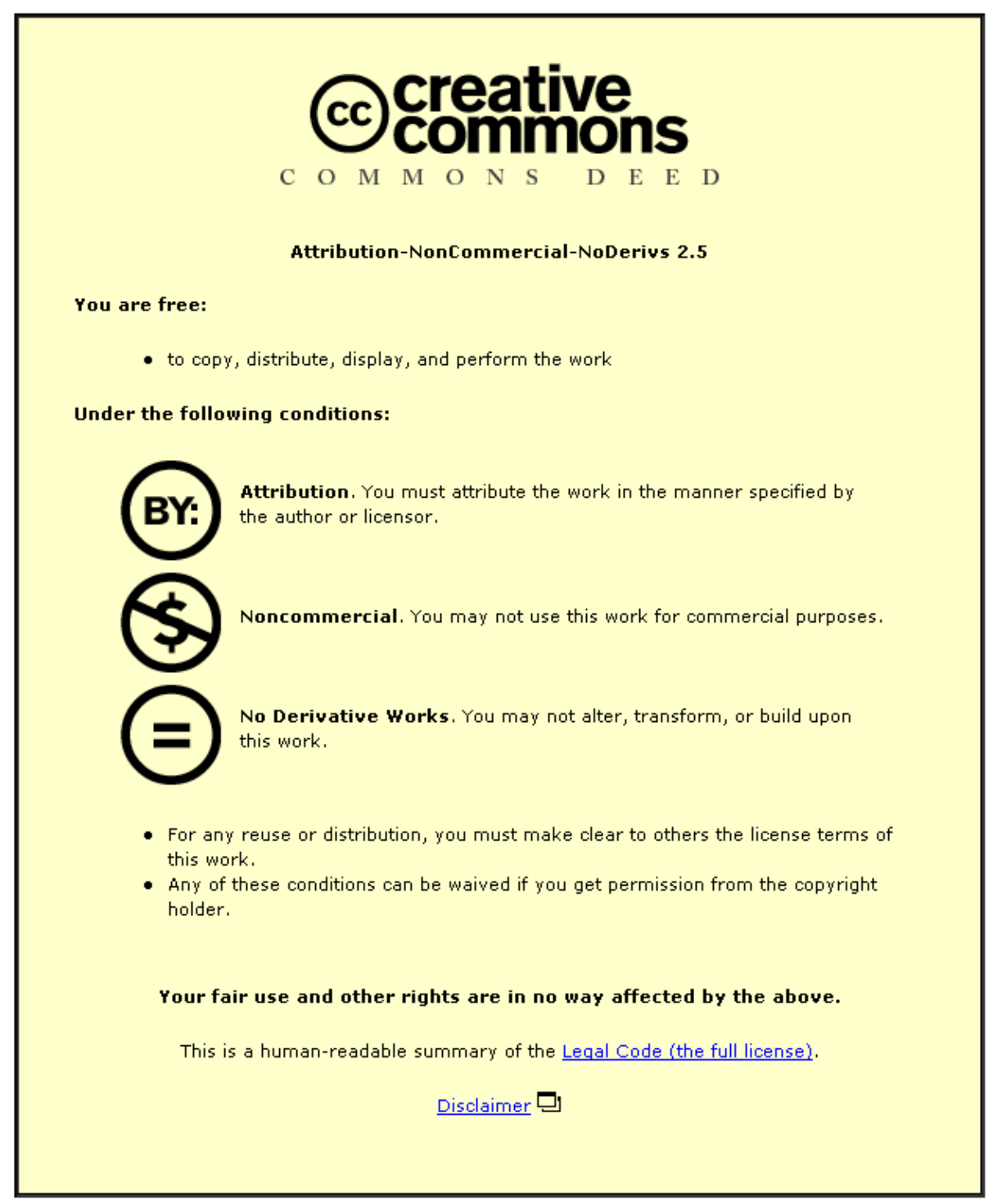

For the full text of this licence, please go to: http://creativecommons.org/licenses/by-nc-nd/2.5/ 


\title{
Use of multiobjective genetic algorithms to optimize inter-vehicle active suspensions
}

T X Mei ${ }^{1 *}$ and $\mathbf{R}$ M Goodall ${ }^{2}$

${ }^{1}$ School of Electronic and Electrical Engineering, The University of Leeds, Leeds, UK

${ }^{2}$ Department of Electronic and Electrical Engineering, Loughborough University, Loughborough, Leicestershire, UK

\begin{abstract}
This paper studies inter-vehicle active suspensions for railway vehicles and presents an optimization process for the design of vertical active suspension controllers using multiobjective genetic algorithms. A three-vehicle train set is used in the study and two active control schemes are considered primarily to provide the best improvement in the passenger ride quality. The first scheme uses only actuators placed between adjacent vehicles while the second adds two actuators between bogie and vehicle body at either end of the train set in addition to the inter-vehicle actuators. The development of the control laws is assisted by the use of genetic algorithms to achieve the 'best' compromise of different design criteria, especially that between the ride quality and the suspension deflections. The study shows that, when the control laws for the proposed active schemes are optimized, a significant improvement in the vertical ride quality on random tracks is obtained and in the mean time the suspension deflections can be kept within their allowed clearance when the vehicles run on to a gradient.
\end{abstract}

Keywords: active suspensions, inter-vehicle, railway, genetic algorithms

NOTATION
$a(1), \ldots, a(9)$
$A_{\mathrm{rv}}$
$c_{\mathrm{r}}$
$d(1), \ldots, d(6)$
$f_{\mathrm{c}}, f_{\mathrm{c} 1}, f_{\mathrm{c} 2}, f_{\mathrm{e}}$
$f_{\mathrm{t}}$
$F_{12}, F_{23}$
$F_{\mathrm{a}}, F_{\mathrm{b}}$
$G_{12}(1), \ldots, G_{12}(6)$
$G_{23}(1), \ldots, G_{23}(6)$
$G_{\mathrm{a}}, G_{\mathrm{b}}$
$k_{\mathrm{a}}$

body accelerations at front suspension, centre and rear suspension of three vehicles track irregularity constant secondary damping (due to the orifice) per bogie $(46.11 \mathrm{kN} \mathrm{s} / \mathrm{m})$ suspension deflections filter cut-off frequencies frequency of the track irregularities inter-vehicle actuators and actuator forces end actuators, and actuator forces control gains for the actuator between vehicle 1 and vehicle 2 control gains for the actuator between vehicle 2 and vehicle 3 control gains for end actuators secondary area stiffness per bogie $(2 \mathrm{~N} / \mathrm{m})$

$k_{\mathrm{r}}$
$k_{\mathrm{s}}$
$I_{\mathrm{v}}$
$L_{\mathrm{f}}$
$L_{\mathrm{v}}$
$m_{\mathrm{v}}$
$n$
$s$
$V_{\mathrm{s}}$
$z(1), \ldots, z(6)$
$z_{\mathrm{a}}(1), \ldots, z_{\mathrm{a}}(6)$
$z_{\mathrm{b}}(1), \ldots, z_{\mathrm{b}}(6)$
$z_{\mathrm{v} 1}, z_{\mathrm{v} 2}, z_{\mathrm{v} 3}$
$\phi_{\mathrm{v} 1}, \phi_{\mathrm{v} 2}, \phi_{\mathrm{v} 3}$

\section{INTRODUCTION}

The use of actively controlled suspensions for railway vehicles has been studied for many years, and it has been secondary reservoir stiffness per bogie $(508 \mathrm{kN} / \mathrm{m})$ secondary airbag stiffness per bogie $(1016 \mathrm{kN} / \mathrm{m})$ vehicle body pitch inertia (2310000 $\mathrm{kg} \mathrm{m}^{2}$ ) half of the vehicle length $(13.5 \mathrm{~m})$ half distance between bogies $(9.5 \mathrm{~m})$ vehicle body mass $(38000 \mathrm{~kg})$ number of vehicle in a train set Laplace (derivative) operator train speed $(80 \mathrm{~m} / \mathrm{s}, 288 \mathrm{~km} / \mathrm{h})$ vertical displacements of train set at suspension mounting positions vertical displacements of air spring midpoints

vertical displacements of the track vertical displacements of the centre position of three vehicles pitch angle of the three vehicles
The MS was received on 12 April 2001 and was accepted after revision for publication on 23 A ugust 2001.

* Corresponding author: School of Electronic and Electrical Engineering, The University of Leeds, Leeds LS2 9JT, UK. 
generally accepted that active suspensions are able to offer substantial improvements beyond what is possible passively [1]. Active tilting controls for increasing train speed on curved tracks without compromising passenger comfort has proved to be extremely successful in service operation [2], and it appears certain that active secondary suspensions will be widely used. Many studies on secondary active control for railway vehicles have so far been based on actuators being placed between the vehicle body and bogies. In principle these can be used as a replacement for the conventional passive suspensions, but in practice they are most likely to provide a supplement in parallel/series with the existing suspensions. A large variety of design approaches has been used for active suspension controllers, and many intuitive and classical approaches are based on the well-known method 'skyhook damping' developed by Karnopp [3]. This control strategy provides damping to an absolute datum and hence achieves high levels of modal damping without increasing the suspension's transmissibility at higher frequencies [1].

This paper studies an active control scheme where actuators are fitted between adjacent vehicles of a train set, rather than the more conventional location under the vehicle body. This active configuration is a natural extension of the passive inter-vehicle dampers that are already installed on the British Rail MkIV, French TGV and the Japanese Shinkansen train sets [4]. Those dampers are primarily used to improve the lateral ride quality, but this paper studies active control in the vertical direction. The arrangement of inter-vehicle active suspensions reduces the number of actuators required and places less restriction on the size of actuators. It also has the advantages of improved reliability because of a lower component count and more favourable environment (less vibration and more space, as they are located above the secondary suspension), as well as lower bandwidth requirement of actuators [5]. This difference in bandwidth is an important factor, because an actuator within the suspension must produce very small forces at high frequency even when there is substantial high-frequency movement across the suspension due to bogie dynamic activity. By contrast at high frequencies there should be negligible movement in an inter-vehicle actuator because both the vehicle bodies should be relatively still in space at high frequencies. However, unlike the more conventional configuration for which control laws can be designed using a single-vehicle model or even a bogie model, the development of controllers for the intervehicle active suspensions is dependent upon the dynamics of the complete train set (being of much higher order) and the design can be much more difficult.

Multiobjective genetic algorithms are used in this study to assist in the control design and to tackle a difficult design conflict between the ride quality and suspension deflection caused by two distinct track inputs. Railway track consists of two different components: deterministic features such as curves and gradients, which form the intended inputs that the vehicle should follow, and stochastic inputs representing the errors from the intended profile, i.e. the track roughness. The suspension designer needs to optimize the ride quality, principally by minimizing the response to the track roughness, because the intended track geometry is designed not to cause discomfort to the passengers. At the same time the maximum suspension deflection must be constrained otherwise excessive accelerations are experienced as the limits of travel are reached, but these maxima are principally associated with the suspension's response to the deterministic features rather than to the track roughness. It is possible to represent the trade-off between ride quality and suspension deflection by means of a quadratic cost function containing a weighted combination of body accelerations and suspension deflections, and then use standard linear quadratic (LQ) optimal controller design. Certainly this procedure is helpful, but it does not meet the requirements mentioned because it is necessary to constrain the suspension deflection, not minimize it. In fact the designer must use the available suspension working space to absorb the high frequency movements of the track and prevent their effect reaching the vehicle body. Also the various track inputs transmitted via the vehicle's wheels are essentially the same input but with different time delays. However, linear quadratic optimal control is only correct when such inputs are uncorrelated. Of course, there are ways of representing these time delays as part of the system model, but it can readily be shown that this is not a practical solution.

Optimization using genetic algorithms (GAs) can deal with the different input types and analysis methods, and the approach can be used to satisfy constraints rather than simply achieve a minimum; also the dynamic complexity of the vehicles, which might cause difficulties with other forms of optimization, can be accommodated. The following sections show how GAs have been used successfully to provide excellent solutions for the active suspensions.

\section{VEHICLE CONFIGURATION AND TRACK CHARACTERISTICS}

The side-view model of a three-vehicle train set used in the study is shown in Fig. 1 to demonstrate the effectiveness of the optimization approach, and the work can be easily extended for a train set with more vehicles. For each vehicle, only the vehicle body and the two secondary suspensions are considered in the model. The secondary suspensions are represented by a linearized airbag model. Primary suspensions and bogies are excluded for simplicity as the main concern here is 


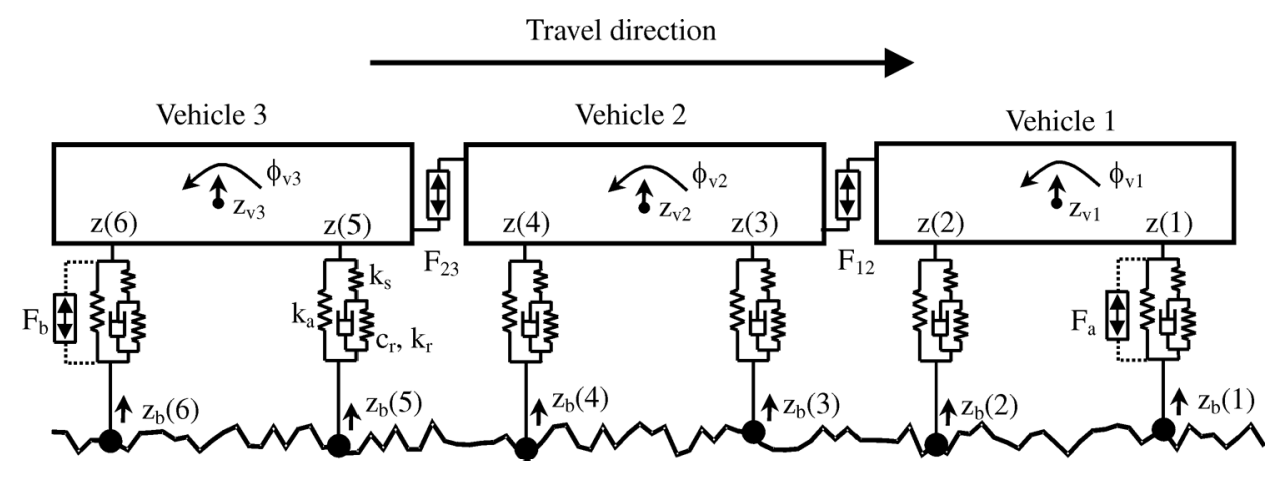

Fig. 1 Train set of three vehicles

the ride quality, which is principally provided by the secondary suspensions. Vehicle parameters are derived from a typical intercity train.

Two ideal actuators (producing forces $F_{12}$ and $F_{23}$ ) are placed between the adjacent vehicles to implement the inter-vehicle active control schemes; this will be studied in detail in Section 5. In addition, it may be sensible to fit two extra actuators (producing forces $F_{\text {a }}$ and $F_{\mathrm{b}}$ ) at the front and rear end of the train set in the usual position, i.e. in parallel with the secondary suspensions (shown in the dotted line), to provide the further improvement of the passenger comfort, as will be demonstrated in Section 6. The general scheme can be extended to a train of $n$ vehicles having a total of $n+1$ actuators, i.e. $n-1$ inter-vehicle actuators and optionally the two actuators at the ends of the train.

When the railway suspensions (passive or active) are designed, the response to the deterministic (the design alignment) and random (track irregularities) inputs of the track must be taken into account. In the study, a typical railway gradient of 1 per cent is assumed with a superimposed acceleration limit of $0.5 \mathrm{~m} / \mathrm{s}^{2}$, a value which is specified for passenger comfort reasons and is used to determine the design alignment of the track. At a typical speed of $80 \mathrm{~m} / \mathrm{s}$ this corresponds to a $1.6 \mathrm{~s}$ transitional section. Normally the track gradient response is not a significant design aspect for vertical passive suspensions, but for active suspensions which are usually based upon 'skyhook' damping large suspension deflections can occur $[\mathbf{1}, \mathbf{6}]$, hence the need to specify the deterministic input to be used in the study.

The random track, representing the roughness of a typical main line, is derived to give an appropriate spatial power spectrum $\left(A_{\mathrm{rv}} / f_{\mathrm{t}}^{2}\right)$ for the track vertical position. This is a simplified version of the generalized power spectrum, which has higher-order terms in the denominator, but for secondary suspension studies the differences are relatively small. Measured track data are used for a particular vehicle design, but given the general nature of this study, the simplified track input form is appropriate.

\section{MODELLING}

As the active suspensions in the vertical direction are studied, only a side-view model of the train set is necessary. There are two degrees of freedom for each vehicle associated with its bounce and pitch motions and a total of six degrees of freedom for the three-vehicle train set. The passive suspension parameters are tuned to obtain good ride quality as far as possible, which give a typical body bounce frequency of $0.8 \mathrm{~Hz}$ with 15 per cent damping and a body pitch mode of $0.9 \mathrm{~Hz}$ with 20 per cent damping. The airbag is a crucial component in the ride performance of a vehicle, and a combination of linear springs and dampers is used to represent its dynamics, as shown in Fig. 1. Although the true behaviour is non-linear, this model is accepted to be reasonably accurate [7]. A mathematical representation of the train set is given in the following equations:

$$
\begin{aligned}
& m_{\mathrm{v}} \ddot{z}_{\mathrm{v} 1}+2\left(k_{\mathrm{a}}+k_{\mathrm{s}}\right) z_{\mathrm{v} 1}-k_{\mathrm{s}} z_{\mathrm{a}}(1)-k_{\mathrm{s}} z_{\mathrm{a}}(2) \\
& \quad \supset k_{\mathrm{a}} z_{\mathrm{b}}(1)+k_{\mathrm{a}} z_{\mathrm{b}}(2)+F_{12}+F_{\mathrm{a}} \\
& I_{\mathrm{v}} \ddot{\phi}_{\mathrm{v} 1}+2\left(k_{\mathrm{a}}+k_{\mathrm{s}}\right) L_{\mathrm{v}}^{2} \phi_{\mathrm{v} 1}-k_{\mathrm{s}} L_{\mathrm{v}} z_{\mathrm{a}}(1)+k_{\mathrm{s}} L_{\mathrm{v}} z_{\mathrm{a}}(2) \\
& \quad \supset k_{\mathrm{a}} L_{\mathrm{v}} z_{\mathrm{b}}(1)-k_{\mathrm{a}} L_{\mathrm{v}} z_{\mathrm{b}}(2)-L_{\mathrm{f}} F_{12}+L_{\mathrm{v}} F_{\mathrm{a}} \\
& c_{\mathrm{r}} \dot{z}_{\mathrm{a}}(1)+\left(k_{\mathrm{s}}+k_{\mathrm{r}}\right) z_{\mathrm{a}}(1)-k_{\mathrm{s}} z_{\mathrm{v} 1}-k_{\mathrm{s}} L_{\mathrm{v}} \phi_{\mathrm{v} 1} \\
& \quad \supset c_{\mathrm{r}} \dot{z}_{\mathrm{b}}(1)+k_{\mathrm{r}} z_{\mathrm{b}}(1) \\
& c_{\mathrm{r}} \dot{z}_{\mathrm{a}}(2)+\left(k_{\mathrm{s}}+k_{\mathrm{r}}\right) z_{\mathrm{a}}(2)-k_{\mathrm{s}} z_{\mathrm{v} 1}+k_{\mathrm{s}} L_{\mathrm{v}} \phi_{\mathrm{v} 1} \\
& \quad \supset c_{\mathrm{r}} \dot{z}_{\mathrm{b}}(2)+k_{\mathrm{r}} z_{\mathrm{b}}(2)
\end{aligned}
$$

$$
\begin{gathered}
m_{\mathrm{v}} \ddot{z}_{\mathrm{v} 2}+2\left(k_{\mathrm{a}}+k_{\mathrm{s}}\right) z_{\mathrm{v} 2}-k_{\mathrm{s}} z_{\mathrm{a}}(3)-k_{\mathrm{s}} z_{\mathrm{a}}(4) \\
\supset k_{\mathrm{a}} z_{\mathrm{b}}(3)+k_{\mathrm{a}} z_{\mathrm{b}}(4)-F_{12}+F_{23}
\end{gathered}
$$




$$
\begin{aligned}
& I_{\mathrm{v}} \ddot{\phi}_{\mathrm{v} 2}+2\left(k_{\mathrm{a}}+k_{\mathrm{s}}\right) L_{\mathrm{v}}^{2} \phi_{\mathrm{v} 2}-k_{\mathrm{s}} L_{\mathrm{v}} z_{\mathrm{a}}(3)+k_{\mathrm{s}} L_{\mathrm{v}} z_{\mathrm{a}}(4) \\
& \quad \supset k_{\mathrm{a}} L_{\mathrm{v}} z_{\mathrm{b}}(3)-k_{\mathrm{a}} L_{\mathrm{v}} z_{\mathrm{b}}(4)-L_{\mathrm{f}} F_{12}-L_{\mathrm{f}} F_{23} \\
& c_{\mathrm{r}} \dot{z}_{\mathrm{a}}(3)+\left(k_{\mathrm{s}}+k_{\mathrm{r}}\right) z_{\mathrm{a}}(3)-k_{\mathrm{s}} z_{\mathrm{v} 2}-k_{\mathrm{s}} L_{\mathrm{v}} \phi_{\mathrm{v} 2} \\
& \quad \supset c_{\mathrm{r}} \dot{z}_{\mathrm{b}}(3)+k_{\mathrm{r}} z_{\mathrm{b}}(3) \\
& c_{\mathrm{r}} \dot{z}_{\mathrm{a}}(4)+\left(k_{\mathrm{s}}+k_{\mathrm{r}}\right) z_{\mathrm{a}}(4)-k_{\mathrm{s}} z_{\mathrm{v} 2}+k_{\mathrm{s}} L_{\mathrm{v}} \phi_{\mathrm{v} 2} \\
& \quad \supset c_{\mathrm{r}} \dot{z}_{\mathrm{b}}(4)+k_{\mathrm{r}} z_{\mathrm{b}}(4) \\
& m_{\mathrm{v}} \ddot{z}_{\mathrm{v} 3}+2\left(k_{\mathrm{a}}+k_{\mathrm{s}}\right) z_{\mathrm{v} 3}-k_{\mathrm{s}} z_{\mathrm{a}}(5)-k_{\mathrm{s}} z_{\mathrm{a}}(6) \\
& \quad \supset k_{\mathrm{a}} z_{\mathrm{b}}(5)+k_{\mathrm{a}} z_{\mathrm{b}}(6)-F_{23}+F_{\mathrm{b}} \\
& I_{\mathrm{v}} \ddot{\phi}_{\mathrm{v} 3}+2\left(k_{\mathrm{a}}+k_{\mathrm{s}}\right) L_{\mathrm{v}}^{2} \phi_{\mathrm{v} 3}-k_{\mathrm{s}} L_{\mathrm{v}} z_{\mathrm{a}}(5)+k_{\mathrm{s}} L_{\mathrm{v}} z_{\mathrm{a}}(6) \\
& \quad \supset k_{\mathrm{a}} L_{\mathrm{v}} z_{\mathrm{b}}(5)-k_{\mathrm{a}} L_{\mathrm{v}} z_{\mathrm{b}}(6)-L_{\mathrm{f}} F_{23}-L_{\mathrm{v}} F_{\mathrm{b}} \\
& c_{\mathrm{r}} \dot{z}_{\mathrm{a}}(5)+\left(k_{\mathrm{s}}+k_{\mathrm{r}}\right) z_{\mathrm{a}}(5)-k_{\mathrm{s}} z_{\mathrm{v} 3}-k_{\mathrm{s}} L_{\mathrm{v}} \phi_{\mathrm{v} 3} \\
& \quad \supset c_{\mathrm{r}} \dot{z}_{\mathrm{b}}(5)+k_{\mathrm{r}} z_{\mathrm{b}}(5) \\
& \quad \supset c_{\mathrm{r}} \dot{z}_{\mathrm{b}}(6)+k_{\mathrm{r}} z_{\mathrm{b}}(6) \\
&
\end{aligned}
$$

Also of concern are the movements of the vehicles at the suspension mounting positions, which may be represented as follows:

$$
\begin{aligned}
& z(1) \supset z_{\mathrm{v} 1}+L_{\mathrm{v}} \phi_{\mathrm{v} 1}, \quad z(2) \supset z_{\mathrm{v} 1}-L_{\mathrm{v}} \phi_{\mathrm{v} 1} \\
& z(3) \supset z_{\mathrm{v} 2}+L_{\mathrm{v}} \phi_{\mathrm{v} 2}, \quad z(4) \supset z_{\mathrm{v} 2}-L_{\mathrm{v}} \phi_{\mathrm{v} 2} \\
& z(5) \supset z_{\mathrm{v} 3}+L_{\mathrm{v}} \phi_{\mathrm{v} 3}, \quad z(6) \supset z_{\mathrm{v} 3}-L_{\mathrm{v}} \phi_{\mathrm{v} 3}
\end{aligned}
$$

\section{PERFORMANCE OF PASSIVE VEHICLES}

The main outputs of interest from the vehicles are the body accelerations at various measurement positions and the secondary suspension deflections. Train performance is evaluated through computer simulations on a time history of both the random and the deterministic track inputs. To assess the ride quality, r.m.s. vertical accelerations experienced at three points of each vehicle are considered: one at the centre of gravity, one above the front secondary suspension and one above the rear secondary suspension. Column P0 of Table 1 gives the r.m.s. accelerations at those positions of the passive train set. As all three vehicles are identical without intervehicle suspensions, the accelerations experienced on each one should be strictly the same and the small differences shown in the table are due to the time delays of the random track used in the simulation for the second and third vehicles. At the vehicle speed of $80 \mathrm{~m} / \mathrm{s}$ $(288 \mathrm{~km} / \mathrm{h})$, the r.m.s. accelerations at the front, centre and rear points of each vehicle are around $0.307,0.255$ and $0.364 \mathrm{~m} / \mathrm{s}^{2}$ respectively, with the rear position being the worst. Also given in the table are the overall r.m.s. values of each vehicle and the entire train. Column P0 of

\begin{tabular}{|c|c|c|c|c|c|c|c|c|c|}
\hline & & \multirow{2}{*}{$\begin{array}{l}\text { Passive } \\
\text { P0 }\end{array}$} & \multicolumn{7}{|c|}{ Active } \\
\hline & & & A 1 & A2 & A3 & A4 & A5 & A6 & A7 \\
\hline \multirow[t]{3}{*}{ Vehicle 1} & Front & 0.307 & 0.292 & 0.309 & 0.297 & 0.296 & 0.053 & 0.260 & 0.243 \\
\hline & Centre & 0.255 & 0.249 & 0.252 & 0.245 & 0.245 & 0.151 & 0.232 & 0.249 \\
\hline & Rear & 0.364 & 0.328 & 0.377 & 0.316 & 0.294 & 0.299 & 0.290 & 0.313 \\
\hline \multirow[t]{3}{*}{ Vehicle 2} & Front & 0.306 & 0.244 & 0.281 & 0.260 & 0.294 & 0.232 & 0.268 & 0.276 \\
\hline & Centre & 0.253 & 0.235 & 0.197 & 0.241 & 0.252 & 0.222 & 0.247 & 0.220 \\
\hline & Rear & 0.361 & 0.338 & 0.267 & 0.337 & 0.329 & 0.329 & 0.385 & 0.332 \\
\hline \multirow[t]{3}{*}{ Vehicle 3} & Front & 0.303 & 0.241 & 0.344 & 0.249 & 0.255 & 0.288 & 0.219 & 0.295 \\
\hline & Centre & 0.252 & 0.242 & 0.267 & 0.245 & 0.256 & 0.146 & 0.211 & 0.204 \\
\hline & Rear & 0.355 & 0.353 & 0.383 & 0.353 & 0.344 & 0.050 & 0.301 & 0.299 \\
\hline \multirow[t]{4}{*}{ Overall } & Vehicle 1 & 0.312 & 0.291 & 0.317 & 0.287 & 0.280 & 0.196 & 0.262 & 0.270 \\
\hline & Vehicle 2 & 0.310 & 0.277 & 0.251 & 0.283 & 0.293 & 0.265 & 0.306 & 0.280 \\
\hline & Vehicle 3 & 0.306 & 0.284 & 0.335 & 0.286 & 0.288 & 0.189 & 0.247 & 0.270 \\
\hline & Train & 0.309 & 0.284 & 0.304 & 0.285 & 0.287 & 0.220 & 0.273 & 0.273 \\
\hline
\end{tabular}
Table 2 gives the maximum suspension deflections of all three vehicles running on to the gradient and on the random track, which are around 43.1 and $46.4 \mathrm{~mm}$ for the front and rear suspensions respectively on the gradient and about $10 \mathrm{~mm}$ less on the random track. It should be noted that the primary suspensions are not included in the model, and therefore both the r.m.s. accelerations on the vehicles and the suspension deflections given in the paper will be somewhat higher than those on real vehicles. However, this should not affect the general validity of the study, as the passive vehicle is used as a basis of comparison and outcomes from active schemes studied in the paper are assessed in relative terms to the results of the passive vehicle.

Table 1 Ride quality on random track (r.m.s. value, $\mathrm{m} / \mathrm{s}^{2}$ ) 
Table 2 Maximum suspension deflections (mm)

\begin{tabular}{|c|c|c|c|c|c|c|c|c|c|c|}
\hline & & & \multirow{2}{*}{$\begin{array}{l}\text { Passive } \\
\text { P0 }\end{array}$} & \multicolumn{7}{|c|}{ Active } \\
\hline & & & & A1 & A2 & A3 & A4 & A5 & A6 & A7 \\
\hline \multirow[t]{6}{*}{ On gradient } & \multirow[t]{2}{*}{ Vehicle 1} & Front & 43.1 & 160 & 40.8 & 42.7 & 42.4 & 2973 & 59.8 & 69.7 \\
\hline & & Rear & 46.4 & 914 & 57.5 & 48.2 & 59.4 & 323 & 56.9 & 42.2 \\
\hline & \multirow[t]{2}{*}{ Vehicle 2} & Front & 43.1 & 981 & 32.8 & 59.4 & 42.4 & 360 & 33.8 & 43.6 \\
\hline & & Rear & 46.4 & 811 & 45.8 & 59.5 & 58.1 & 404 & 58.5 & 58.5 \\
\hline & \multirow[t]{2}{*}{ Vehicle 3} & Front & 43.1 & 647 & 42.4 & 43.8 & 35.9 & 328 & 42.1 & 39.1 \\
\hline & & Rear & 46.4 & 149 & 48.6 & 48.2 & 48.9 & 2953 & 59.7 & 70.0 \\
\hline \multirow[t]{6}{*}{ On random track input } & \multirow[t]{2}{*}{ Vehicle 1} & Front & 32.5 & 32.7 & 33.0 & 30.9 & 31.8 & 69.4 & 36.9 & 38.0 \\
\hline & & Rear & 35.7 & 49.5 & 38.5 & 39.1 & 33.0 & 32.9 & 35.5 & 46.0 \\
\hline & \multirow[t]{2}{*}{ Vehicle 2} & Front & 32.5 & 39.5 & 26.2 & 31.1 & 32.0 & 30.9 & 27.9 & 33.7 \\
\hline & & Rear & 35.5 & 46.9 & 31.6 & 38.9 & 35.2 & 37.4 & 40.7 & 44.2 \\
\hline & \multirow[t]{2}{*}{ Vehicle 3} & Front & 32.4 & 35.3 & 35.6 & 28.2 & 30.7 & 34.1 & 26.1 & 38.6 \\
\hline & & Rear & 35.5 & 35.4 & 38.5 & 37.0 & 37.0 & 69.0 & 39.6 & 46.0 \\
\hline
\end{tabular}

\section{ACTIVE CONTROL WITH INTER-VEHICLE ACTUATORS ONLY}

As stated earlier, this paper studies the use of intervehicle active control for achieving improvements in ride quality rather than other more conventional structures. This is a relatively new, unfamiliar idea and very little has been studied for its control laws, probably because of the complexity involved. In reference [5], two control schemes have been studied. One takes the approach of optimal control. In addition to the reasons already stated, this solution is not ideal because it requires full state feedback and the order of the controller can be very high (increases with the number of vehicles). The other scheme implements the principle of absolute damping to improve the ride quality of the centre vehicle of a three-vehicle train set, and complementary filters are used to reduce the actuator deflections. The control design is simplified by using only the local measurements (bounce and pitch velocities from the centre vehicle and actuator deflections) as feedback for the controllers.

This study adopts the concept of 'skyhook damping' for the inter-vehicle active controllers with the aim of improving the passenger ride comfort on all vehicles. Figure 2 shows the control structure. Two measurements from each vehicle body at the positions of the front and rear suspensions, making a total of six measurements, are used for the two controllers controlling the two inter-vehicle actuators. Two additional controllers ( $\mathrm{a}$ and $\mathrm{b}$ ) indicated by dotted lines are used for the end actuators and will be discussed in the next section. The measurements required for a skyhook damping strategy are the vertical velocities of the vehicles, but in practice accelerometers will be used and signals integrated to give the required information.

Genetic algorithms (GAs) are used in this study to decide the parameters of the two controllers to obtain the best performance possible within specified constraints. A GA is a stochastic global search method that mimics the process of natural biological evolution. GAs operate on a population of potential solutions by applying the principle of survival of the fittest to produce better and better approximations to a solution [8]. Figure 3 shows a typical GA searching process. One of the most important issues in the use of GAs is the definition of the objective functions, which will dominate the way the control gain is to be selected. Several cases are studied and discussed as follows.

\section{Case A 1: overall r.m.s. acceleration only}

The r.m.s. accelerations are often used as a measure of ride comfort, and it is therefore sensible to define the overall r.m.s. acceleration of the train set (i.e. the r.m.s. value of all nine r.m.s. accelerations from the three vehicles) as the objective as shown in the following equation:

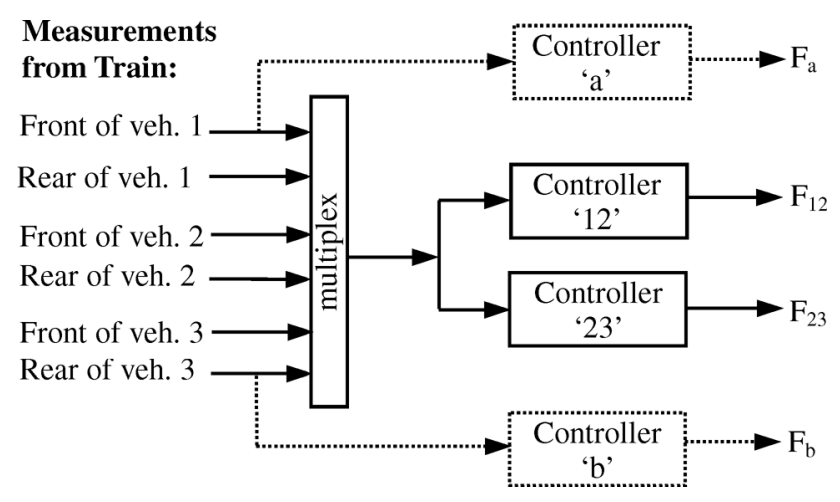

Fig. 2 Control structure 


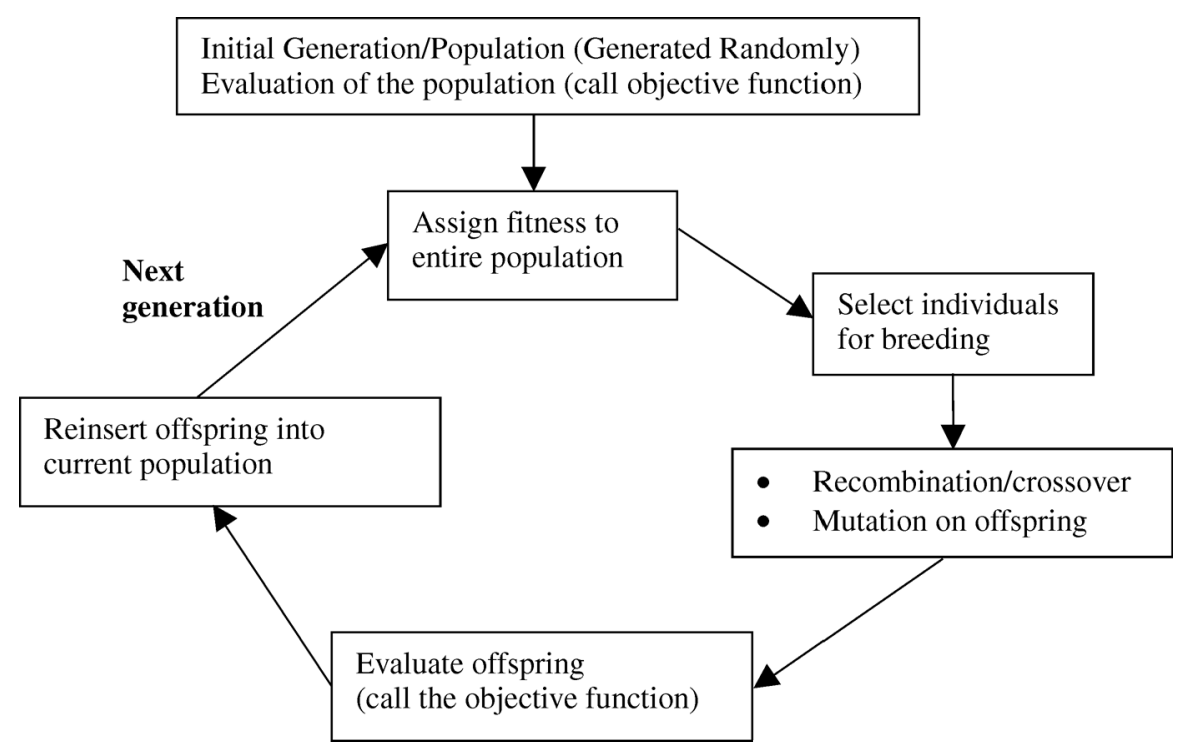

Fig. 3 A typical GA searching process

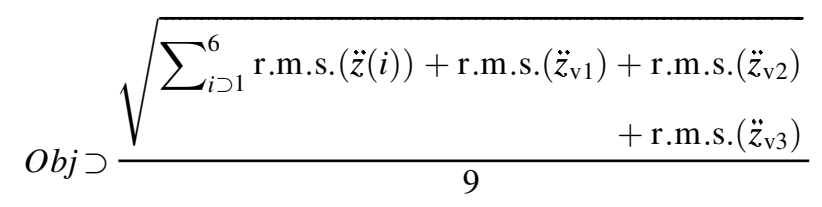

Positions at the centre of a vehicle and above the secondary suspensions are most commonly used to provide a direct measure of ride quality, and therefore the accelerations at those positions are explicitly defined in the objective, even though one of three accelerations of each vehicle can be derived from the other two.

In this first case, the suspension deflection requirement is excluded in order to examine what could be achieved without other constraints. Ideal velocity feedback is assumed in this case and 12 skyhook damping gains are to be optimized by the GA for the two controllers as defined by ( $F_{\mathrm{a}}$ and $F_{\mathrm{b}}$ are set to 0$)$

$$
\begin{aligned}
& F_{12} \supset \sum_{i \supset 1}^{6} G_{12}(i) \dot{z}(i) \\
& F_{23} \supset \sum_{i \supset 1}^{6} G_{23}(i) \dot{z}(i)
\end{aligned}
$$

The strategy allows each actuator force to be dependent upon any or all of the velocity measurements if required.

Column A 1 of Table 1 gives r.m.s. accelerations of the train set, with the active controllers tuned by the GA, on the random track. The overall r.m.s. acceleration is reduced by 7.9 per cent compared with the passive vehicle (from 0.309 to 0.284 ), with the front ends of the vehicles 2 and 3 benefitting the most (around 20 per cent improvement). Overall, the improvement for the second vehicle (10.8 per cent) is better than that for the vehicles at either end (6-7 per cent), obviously because the vehicles are influenced by both actuators. Notice that even the front end of vehicle 1 and the rear end of vehicle 3 are improved, even though the only actuator is at the other ends of these vehicles. However, the maximum travel distances of the vehicle secondary suspensions are unacceptably large $(149-981 \mathrm{~mm})$ on gradient, as shown in column A1 of Table 2. This is not surprising, because it is known that the 'skyhook damping' concept creates large suspension deflections on deterministic features such as gradients and curves.

\section{Case A2: overall r.m.s. acceleration as objective with suspension deflections constrained}

To reduce the suspension deflections, extra measures need to be introduced into the GA searching process. On the other hand, the suspensions must be allowed to move within their travel space to filter out highfrequency vibrations caused by the track as much as possible and therefore the deflections should not be minimized. With multiobjective GAs, all six suspension deflections are specified as constraints, as given by

$$
d(i) \supset \max \left[\operatorname{abs}\left(z(i)-z_{\mathrm{b}}(i)\right)\right], \quad \text { where } i \supset 1,2, \ldots, 6
$$

and are limited to the maximum design distance (an extra $14 \mathrm{~mm}$ is added to the maximum deflection of the passive vehicle in this case), while the overall r.m.s. acceleration of the train set is still defined as an objective to minimize. Again the same control law using ideal velocity feedback is assumed, and the GA program is rerun to find a new set of 12 control gains in equations (14) and (15). 
The results of the new search are shown in column A2 of Table 1. An improvement of 19.0 per cent is achieved for vehicle 2 compared with the passive train set, with the best improvement of 26.1 per cent at the rear end. However, the ride quality on vehicles 1 and 3 has actually worsened by approximately 1.6 and 9.3 per cent respectively. The suspension deflections are effectively constrained, which are within the maximum value specified.

\section{Case A3: use of high-pass filters and acceleration feedback}

The previous two cases have highlighted the design trade-off between the ride quality on random track and the suspension deflection on deterministic track. Although it is difficult to distinguish the two track features, it is important to recognize that the deterministic track input contains primarily the low-frequency components and the random input has a spectrum across the frequency domain. A practical solution is to add high-pass filters at the feedback point so that the actuators will only react to high-frequency components of the track excitation. The structure of the two controllers with a first order high-pass on each channel, where accelerometers are used to measure the vibrations and the pure integrator is used to produce the velocity signals required for skyhook damping, are defined by the following equations:

$$
\begin{aligned}
& F_{12} \supset \frac{s}{s+2 \pi f_{\mathrm{c} 1}} \frac{1}{s} \sum_{i \supset 1}^{6} G_{12}(i) \ddot{z}(i) \\
& F_{23} \supset \frac{s}{s+2 \pi f_{\mathrm{c} 2}} \frac{1}{s} \sum_{i \supset 1}^{6} G_{23}(i) \ddot{z}(i)
\end{aligned}
$$

In practice the high-pass filters and the integrators would be combined, but they are shown separately to clarify the conceptual approach.

The GA objectives remain the same as in case A2, i.e. to minimize the overall r.m.s. acceleration with all the suspension deflections constrained. In addition to searching for the twelve 'optimal control gains', the GA is also used to select the best cut-off frequencies $\left(f_{\mathrm{c} 1}\right.$, $\left.f_{\mathrm{c} 2}\right)$ for the high-pass filters. Column A3 of Tables 1 and 2 gives the outcomes from the search.

Overall the ride quality of the train is improved by about 8 per cent, with the improvement on three vehicles being 7.9, 8.9 and 6.4 per cent respectively. The most significant improvements are achieved at the rear end of vehicle 1 (13.2 per cent), front end of vehicle 2 (15.1 per cent) and front end of vehicle 3 (17.8 per cent). The least improvements are obtained at the either end of the train set, which is expected as the actuators have less effect on those positions. The maximum suspension deflections are within the specified travel space, but those of the vehicle in the centre are pushed to the limit.

The cut-off frequencies of the two high-pass filters in the controllers are found to be $f_{\mathrm{c} 1} \supset 14.6 \mathrm{~Hz}$ and $f_{\mathrm{c} 2} \supset 10.8 \mathrm{~Hz}$. The frequencies are higher than expected [9], and in fact when combined with the pure integrator the filters become low-pass ones with cut-off frequencies at 14.6 and $10.8 \mathrm{~Hz}$ respectively. This suggests that the inter-vehicle active schemes actually use acceleration signals below the low frequencies in order to achieve the best compromise between ride quality and suspension deflections. This is a significant result because it seems that for inter-vehicle actuators a skyhook strategy may not be the most appropriate- the effect of acceleration feedback will be to affect the suspension stiffness in some manner.

It is worth noting that the ride quality improvement obtained in this case is very close to that in case A1, where no constraints on the suspension deflections were applied. In fact a similar improvement is achieved at all individual positions throughout the train set between cases A1 and A3, as clearly illustrated in Table 1. This indicates that a near-optimal solution is obtained in this case.

\section{Case A4: more complex control structures}

For the active schemes with actuators fitted in the position of secondary suspensions, it has been proved that some more advanced control structures such as the use of complementary filters can offer further performance improvement, and some of the control strategies can be adapted for use within the intervehicle active scheme. The complementary filter requires additional measurement of the suspension deflections, and the controller is structured such that the variation of the cut-off frequency will not affect the system stability. Also studied is a modal control approach where the bounce and pitch modes are decoupled and the controllers are then tuned separately. However, neither of the two more complex control structures appears to offer better performance for the inter-vehicle active scheme. Column A4 of Tables 1 and 2 gives the outcome for the complementary filter approach, which is no better than previous cases.

\section{ACTIVE CONTROL WITH INTER-VEHICLE AND END ACTUATORS}

As has been demonstrated in the previous section, the active control scheme using only the inter-vehicle actuators has a greater influence on ride quality at the positions where the actuators are fitted and a much smaller effect at either end of the train set. It is therefore 
sensible to consider two additional actuators at the front end of the first vehicle and the rear end of the last vehicle, as shown by the dotted lines in Fig. 1 (actuator forces $F_{\mathrm{a}}$ and $F_{\mathrm{b}}$ ). For a train set with more vehicles, the reduction of the number of actuators can still be substantial with this arrangement. The control structures for the original inter-vehicle actuators remain the same, but two additional controllers using local feedback measurements (as shown by the dotted lines in Fig. 2) are used to control the actuators $F_{\mathrm{a}}$ and $F_{\mathrm{b}}$.

\section{Case A 5: pure skyhook damping}

This is very similar to the case A1, and the aim is to find the limit of the scheme on the ride quality improvement without any other constraints. The GA is used to tune 14 control gains, 12 of which are gains for the actuators $F_{12}$ and $F_{23}$, as given in equations (14) and (15), and the remaining two are the skyhook damping gains for the actuators $F_{\mathrm{a}}$ and $F_{\mathrm{b}}$, as specified in the following equations:

$$
\begin{aligned}
& F_{\mathrm{a}} \supset G_{\mathrm{a}} \dot{z}(1) \\
& F_{\mathrm{b}} \supset G_{\mathrm{b}} \dot{z}(6)
\end{aligned}
$$

Ideal velocity feedback is assumed and the only objective specified is the overall r.m.s. acceleration of the train set shown in equation (13). The searching results are given in column A5 of Tables 1 and 2 .

In this case, an overall reduction of almost 30 per cent in body acceleration is achieved, with the improvement on the end vehicles being around 37 per cent and on the central vehicle around 14.6 per cent. The most significant reductions (more than 80 per cent) are obtained at the positions of the two additional actuators. This is, of course, not possible in practice for a number of reasons, not least because it would cause a massive suspension movement of almost $3 \mathrm{~m}$.

\section{Case A6: use of high-pass filter and acceleration feedback}

To reduce the suspension deflections, four high-pass filters are used for the four actuators respectively and acceleration signals are now used instead of the ideal velocity feedback. A total of 16 parameters are to be optimized by the GAs. There are six gains for each of the inter-vehicle controllers and two gains for the end controllers. For simplicity, two cut-off frequencies $\left(f_{\mathrm{c}}\right.$ and $f_{\mathrm{e}}$ ) are specified for inter-vehicle and end actuators. The controllers for the four actuators are specified by the following equations:

$$
\begin{aligned}
& F_{12} \supset \frac{s}{s+2 \pi f_{\mathrm{c}}} \frac{1}{s} \sum_{i \supset 1}^{6} G_{12}(i) \ddot{z}(i) \\
& F_{23} \supset \frac{s}{s+2 \pi f_{\mathrm{c}}} \frac{1}{s} \sum_{i \supset 1}^{6} G_{23}(i) \ddot{z}(i) \\
& F_{\mathrm{a}} \supset \frac{s}{s+2 \pi f_{\mathrm{e}}} \frac{1}{s} G_{\mathrm{a}} \ddot{z}(1) \\
& F_{\mathrm{b}} \supset \frac{s}{s+2 \pi f_{\mathrm{e}}} \frac{1}{s} G_{\mathrm{b}} \ddot{z}(6)
\end{aligned}
$$

The searching objective is still the overall r.m.s. acceleration of the train set and the constraints are the maximum suspension deflections as given in equations (13) and (16) respectively.

Column A6 in Tables 1 and 2 gives the results from the GA optimization procedure. The overall ride quality is improved by about 12 per cent compared with the passive train. At individual positions, substantial improvements are achieved for all measuring positions on vehicle $1(15.3,8.9$ and 20.2 per cent) and vehicle 3 (27.8, 16.3 and 15.2 per cent) as well as the front end of vehicle 2 (12.5 per cent). However, only a small reduction in the acceleration at the central position of vehicle 2 is obtained, and there is even an increase at the rear end.

The central position of vehicle 2 is not a cause for concern as the r.m.s. acceleration is relatively low $\left(0.247 \mathrm{~m} / \mathrm{s}^{2}\right)$ compared to that at other positions. However, the deterioration in ride quality at the end of the vehicle is not desirable. The likely cause is that the constraint imposed on the suspension deflections affects severely the selection of the control gains and consequently some sacrifices have to be made.

\section{Case A 7: minimization of r.m.s. acceleration of individual vehicles}

The control structure used in this case is exactly the same as that in case A6, but several changes are made in the GA searching process. The overall r.m.s. acceleration of the train set is replaced by the overall accelerations of the three vehicles as three independent objectives, as shown in the following equations:

$$
\begin{aligned}
& \text { Obj1 } \supset \sqrt{\frac{\text { r.m.s. }\left(\ddot{z}_{\mathrm{v} 1}\right)+\text { r.m.s. }(\ddot{z}(1))+\text { r.m.s. }(\ddot{z}(2))}{3}} \\
& \text { Obj2 } \supset \sqrt{\frac{\text { r.m.s. }\left(\ddot{z}_{\mathrm{v} 2}\right)+\text { r.m.s. }(\ddot{z}(3))+\text { r.m.s. }(\ddot{z}(4))}{3}}
\end{aligned}
$$




$$
O b j 3 \supset \sqrt{\frac{\text { r.m.s. }(\ddot{z} \mathrm{v} 3)+\text { r.m.s. }(\ddot{z}(5))+\text { r.m.s. }(\ddot{z}(6))}{3}}
$$

The accelerations of vehicles 1 and $3(O b j 1$ and $O b j 3)$ are defined as constraints (at $0.27 \mathrm{~m} / \mathrm{s}^{2}$ ) and that of the vehicle 2 is defined as the main objective to be minimized. This is because the previous search did not achieve a good result for vehicle 2 . By setting constraints on the r.m.s. acceleration for the end vehicles, it is more likely to achieve a similar and average improvement on ride quality for all vehicles throughout the train set. The constraint on the suspension deflections in equation (16) is also relaxed by another $10 \mathrm{~mm}$, as the former appears to be too tight for the active configuration. The relaxation of the constraint will affect the outcome of the optimization and hence the controller, which in this case is to illustrate that it is sometimes necessary to allow for a larger suspension in order to achieve the full potential of the active control approach.

Column A7 in Tables 1 and 2 presents the results from the optimization process. The overall ride quality of the train set is similar to that achieved in the previous case, but vehicle 2 delivers much improved passenger comfort (9.9, 13.2 and 8.2 per cent at front, centre and rear positions respectively). This is, of course, obtained with a small performance sacrifice for the end vehicles, but this is a preferred result as all the vehicles of the train set now have a similar level of ride quality.

\section{SIMULATIONS AND EVALUATIONS}

From studies above, it is clear that the issue of suspension deflections is an important one in the development of active control strategies. In most cases, the suspension deflections remain largely unchanged compared with passive on random track input, as shown in Table 2. However, if no consideration is given to the design of active controllers, the problem becomes apparent when vehicles travel onto a deterministic track (i.e. a gradient), as demonstrated in cases A1 and A5 in Table 2. Restricting the travel of suspensions is a necessary requirement for the practical implementation of any proposed active control schemes. On the other hand, as the findings in the paper indicate, some relaxation of the maximum suspension deflection will be required for the active controls in order to achieve the desired improvement of ride quality. Therefore a compromise has to be made between the two issues.

The active cases A3, A6 and A7 are of practical interest and Table 3 gives the final 'optimal' control gains obtained in the study. Figure 4 compares the overall ride qualities (normalized by the passive case) between the passive case $\mathrm{P} 0$ and the active cases A3, A6 and A7 (for which the constraint on suspension deflections is applied), while Fig. 5 shows the normalized ride qualities at the front, centre and rear positions on the three vehicles of the train set. If only inter-vehicle actuators are used (case A3), the overall improvement is about 8 per cent compared to the passive vehicle and most gains are obtained at the positions near to the actuators. When two extra actuators are added at the front position of first vehicle and rear end of last vehicle, caution should be given to the design of control gains. In case A6 where only the overall ride quality is used in the optimization, an overall improvement about 12 per cent is achieved, but the ride quality at the rear end of the vehicle in the middle is actually worsened. By defining searching conditions for the GA optimization differently (case A7), a more even distribution of improvements in ride quality is achieved.

Simulation results are also evaluated in the study. Figure 6 gives the time history of accelerations of the passive and active (A6) vehicles at the front position of vehicle 3 on the random track, where the improvement of the active approach is significant. Also, Fig. 7 demonstrates how the suspension deflections are affected by the active control when the train set is running onto a gradient. All suspension deflections are below the predefined limit, obviously because this has been taken into account by the GA optimization when deciding the control gains.

The power requirement of the actuators studied is fairly low. For the active control scheme A6, the r.m.s. value of the actuator power is less than $60 \mathrm{~W}$ with a peak value of $300 \mathrm{~W}$. The maximum actuator force required is $6 \mathrm{kN}$ on both random and deterministic tracks.

Table 3 Control parameters [unit of gains, $\mathrm{MN} /(\mathrm{m} / \mathrm{s})$; unit of frequency, $\mathrm{Hz}$ ]

\begin{tabular}{|c|c|c|c|c|c|c|c|c|}
\hline \multicolumn{3}{|c|}{ A3 } & \multicolumn{3}{|c|}{ A6 } & \multicolumn{3}{|c|}{ A7 } \\
\hline$G_{12}(i)$ & $G_{23}(i)$ & Others & $G_{12}(i)$ & $G_{23}(i)$ & Others & $G_{12}(i)$ & $G_{23}(i)$ & Others \\
\hline 0.48 & -0.42 & $f_{\mathrm{c} 1} \supset 14.6$ & 0.12 & -0.44 & $G_{\mathrm{a}} \supset-0.41$ & 1.93 & -1.52 & $G_{\mathrm{a}} \supset-1.11$ \\
\hline-1.98 & 0.36 & $f_{\mathrm{c} 2} \supset 10.8$ & -1.54 & -0.75 & $G_{\mathrm{b}} \supset-0.29$ & -3.64 & -0.41 & $G_{\mathrm{b}} \supset-0.95$ \\
\hline 2.28 & 0.01 & & 1.40 & 0.95 & $f_{\mathrm{c}} \supset 15.5$ & 1.92 & 1.72 & $f_{\mathrm{c}} \supset 19.9$ \\
\hline 0.01 & -1.39 & & -0.10 & -0.88 & $f_{\mathrm{e}} \supset 9.2$ & 0.73 & -2.16 & $f_{\mathrm{e}} \supset 11.7$ \\
\hline-0.37 & 1.87 & & 0.43 & 2.14 & & 0.38 & 2.95 & \\
\hline 0.14 & -0.32 & & -0.42 & -0.86 & & -1.42 & -1.07 & \\
\hline
\end{tabular}




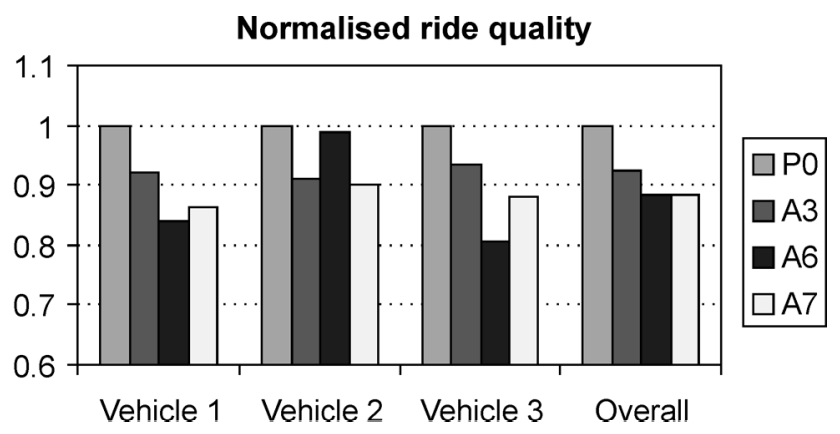

Fig. 4 Comparison of overall ride qualities

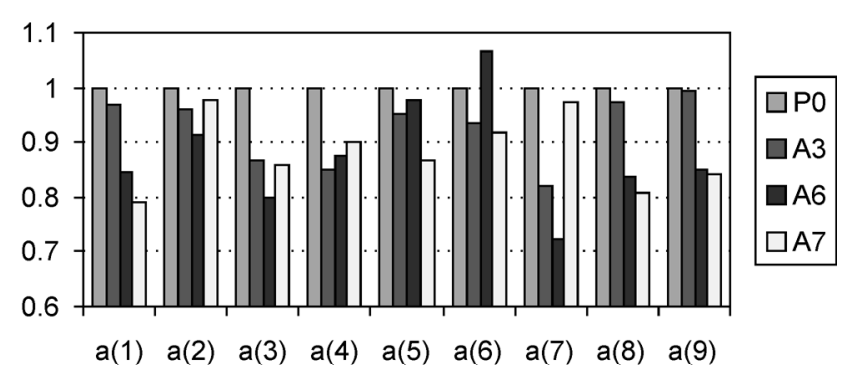

Fig. 5 Comparison of ride qualities at individual points

This paper has addressed only one particular application of the GA optimization, and GAs may be used in many other applications to find 'optimal solutions'. However, it should be noted that, although in theory GAs should always be able to produce the global 'optimal' solution if the searching process is run long enough, in practice this is not always guaranteed and the convergence can sometimes be slow. At present there are no proven methods available to indicate whether a GA search has achieved its optimum. However, some

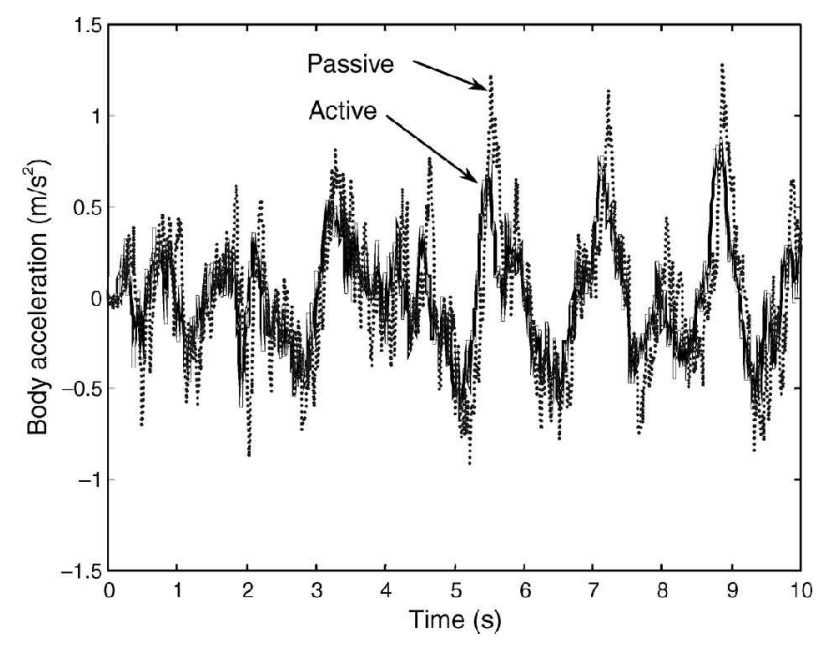

Fig. 6 Body acceleration (passive and active A6)

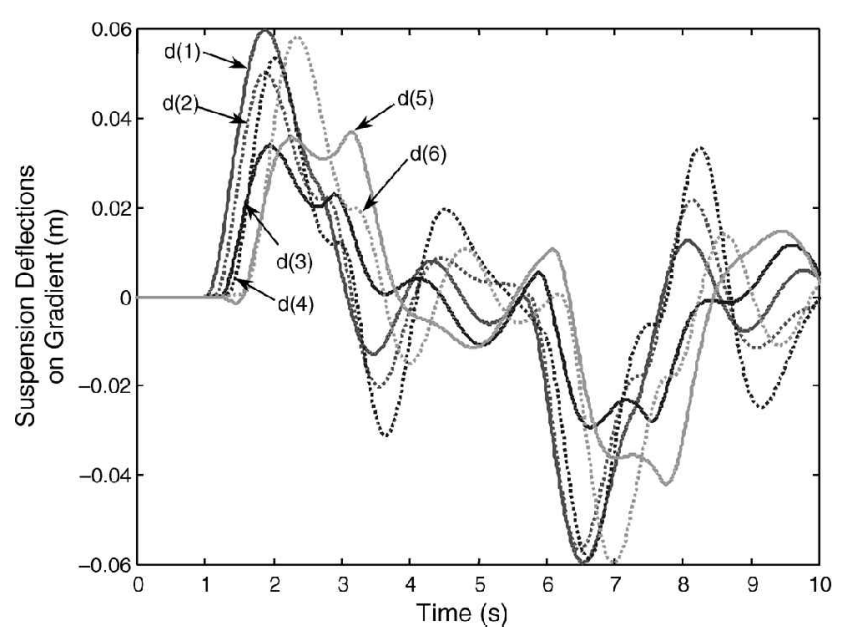

Fig. 7 Suspension deflections (active scheme A6)

practical steps can be taken to maximize the possibility of finding the global optimal solutions, e.g. it may help the searching process when the objectives are re-defined in a different form.

\section{CONCLUSIONS}

This paper has studied active inter-vehicle control strategies for trains. Two control schemes have been investigated: one uses only the inter-vehicle actuators and the other is complemented by two additional actuators between the vehicle body and the bogie at either end of a train set. Multiobjective genetic algorithms have been used extensively in the study for the optimal design of control laws and control gains, where different objectives and constraints have been applied. It has been demonstrated that, although the dynamics of a train set is complex because of its high order and interactions, the design of the active controllers has been made much simpler by the GA optimization procedure.

It has been shown that it is possible to improve the ride quality on the railway vehicles on random track and at the same time to maintain the suspension deflections below an acceptable level on deterministic track input. This has been achieved with the use of a very simple sensing requirement and control structure, which will make the practical implementation of the proposed schemes much easier. There are only two accelerometers required for each vehicle and each controller consists of a set of control gains and a simple first-order filter.

Although the configuration of inter-vehicle actuators does not seem to offer as much improvement on the ride quality as the more conventional scheme that has actuators across or within the secondary suspension, its advantages are also clear. It requires fewer actuators 
(about half of that required for under-body actuators) and has less restriction on the size of actuators. It also has the advantage of improved reliability as well as a lower bandwidth requirement for the actuators, and for this reason offers a practical alternative for active secondary suspension control that could usefully be studied experimentally in order to validate some of the ideas and strategies that have been developed.

Further practical work would involve deriving a better definition of actuator requirements, modelling of the actuator dynamics and assessing the sensitivity of the control laws, in particular to variations in train speed.

\section{ACKNOWLEDGEMENTS}

The authors wish to acknowledge the support of the European Community for funding the project BE974387 and that of the University of Sheffield (Department of Automatic Control and Systems Engineering) for providing the MOGA toolbox used in the study.

\section{REFERENCES}

1 Goodall, R. M. Active railway suspensions: implementation status and technological trends. Veh. Syst. Dynamics, 1997, 28, 87-117.

2 Ford, R. Tilt no longer a novelty in Europe. Railway Gazette Int., April 1997, 249-252.

3 Karnopp, D. C. Active and passive isolation of random vibration. In Isolation of Mechanical Vibration Impact and Noise (Eds J. C. Snowdon and E. E. Ungar), 1973, ASME Monograph, AMD Vol. 1, No. 1, pp. 357-366.

4 Fujimoto, H. and Miyamoto, M. Lateral vibration and its decreasing measure on a Shinkansen train. Proceedings of the 14th IAVSD Symposium on Dynamics of Vehicles on Roads and Tracks, Ann Arbor, Michigan, 1995, pp. 40-42.

5 Pratt, I. Active suspension applied to railway trains. PhD thesis, Loughborough University, 1996.

6 Li, H. and Goodall, R. M. Linear and non-linear skyhook damping control laws for active railway suspensions. Control Engng Practice, 1999, 7, 843-850.

7 Williams, R. A. A comparison of classical and optimal active suspension control systems. PhD thesis, Loughborough University, 1986.

8 Goldberg, D. E. Genetic Algorithms in Search, Optimization and Machine Learning, 1989 (Addison Wesley, London). 